FORMATION Formation emploi

Revue française de sciences sociales

117 | janvier-mars 2012

Enseignement supérieur : les défis de la professionnalisation

\title{
Jeunes diplômés en sciences : les déterminants de l'accès à la recherche-développement privée
}

Young science graduates: determining factors for access to private research and development

Wissenschaftliche hochschulabsolventen: Was ist für den zugang zur privaten forschung und entwicklung ausschlaggebend?

Jóvenes graduados en ciencias: los determinantes del acceso al sector de investigación y desarrollo privado

\section{Claire Bonnard}

\section{(2) OpenEdition}

Journals

Édition électronique

URL : http://journals.openedition.org/formationemploi/3520

DOI : 10.4000/formationemploi.3520

ISSN : 2107-0946

Éditeur

La Documentation française

Édition imprimée

Date de publication : 23 avril 2012

Pagination : 49-68

ISSN : 0759-6340

Référence électronique

Claire Bonnard, « Jeunes diplômés en sciences : les déterminants de l'accès à la recherchedéveloppement privée », Formation emploi [En ligne], 117 | janvier-mars 2012, mis en ligne le 06 juin 2012, consulté le 30 octobre 2020. URL : http://journals.openedition.org/formationemploi/3520 ; DOI : https://doi.org/10.4000/formationemploi.3520 


\title{
Jeunes diplômés en sciences: les déterminants de l'accès à la recherche-développement privée
}

\author{
Claire BONNARD \\ Attachée temporaire d'enseignement et de recherche en sciences économiques à l'IREDU \\ (Institut de recherche sur l'éducation) - université de Bourgogne
}

Résumé

Jeunes diplômés en sciences : les déterminants de l'accès à la recherchedéveloppement privée

L'accès des jeunes diplômés à la $\mathrm{R} \& \mathrm{D}$ (recherche et développement) privée est souvent présenté comme un enjeu majeur pour la compétitivité des pays. Nous analysons ici les déterminants de l'accès et des débuts de carrières dans la $R \& D$ en France. Nous utilisons les données de l'enquête nationale du Céreq de 2007 sur la "Génération 2004 ", et un modèle de régression endogène à régime pour corriger un éventuel biais de sélection. Ainsi, le niveau de diplôme, et notamment l'accès au doctorat, est déterminant dans l'accès et la rémunération en $\mathrm{R} \& \mathrm{D}$ privée. En revanche, le doctorat ne procure aucun avantage salarial dans les autres secteurs. Pour les docteurs, l'expérience antérieure en entreprise, durant la thèse, est décisive, alors que pour les ingénieurs, seule la catégorie d'école d'ingénieurs joue un rôle. Enfin, pour les docteurs en sciences, en début de carrière, et contrairement aux diplômés d'écoles d'ingénieurs, la R\&D est moins rémunératrice que les autres activités.

Mots clés : recherche-développement $\bullet$ premier emploi $\bullet$ enquête d'insertion $\bullet$ doctorat - science pure

Abstract

Young science graduates: determining factors for access to private research and development

Young graduates' access to private $\mathrm{R} \& \mathrm{D}$ (research and development) is often presented as a key issue in a nation's competitiveness. Here, we analyse the determining factors regarding access to and early careers in $\mathrm{R} \& \mathrm{D}$ in France. We use data from the Céreq's 2007 national survey covering the "Generation of 2004" and an endogenous switching regression model to adjust for any possible selection bias. We find that qualification level and, in particular, access to $\mathrm{PhD}$ research, plays a determining role in access private $\mathrm{R} \& \mathrm{D}$ and earnings. In contrast, holding a $\mathrm{PhD}$ does not lead to any advantage in terms of salary in other sectors. One decisive factor is the fact that $\mathrm{PhD}$ graduates have prior experience, during their thesis research, of working in companies, whereas for Engineers, there is a 
significant difference only for the category of Engineering School graduates. Lastly, for a Doctor of Sciences, at the beginning of his or her career, and unlike Engineering School graduates, $\mathrm{R} \& \mathrm{D}$ pays less than other activities.

Key words: research $\&$ development $\bullet$ first-time employment $\bullet$ school-to-work transition survey $\bullet \mathrm{PhD} \bullet$ pure science

Journal of Economic Literature: $\mathrm{O}$ 32, J 24

Traduction : Provence Traduction

La connaissance est considérée comme une ressource cruciale des entreprises. Ainsi, la façon d'attirer et de retenir les scientifiques et les ingénieurs revêt une importance capitale (Manolopoulos, 2006). En 2006, avec plus de 211000 chercheurs en équivalent temps plein, la France se situe au $6^{\text {ème }}$ rang mondial et à la seconde position au niveau de l'Union européenne (derrière l'Allemagne). En revanche, même si, depuis 1985, le nombre de chercheurs dans le privé a triplé, passant de 35100 à plus de 108 000, la France accuse encore un retard important par rapport aux autres pays. Depuis les années 60, la pénurie d'ingénieurs et de scientifiques est souvent évoquée dans les médias. Elle est sujette à de nombreux débats dans la littérature académique, que ce soit aux États-Unis (Butz et al., 2003 ; Brown et al., 2008) ou au sein de l'Union européenne (Gago, 2004, Becker, 2010). De nombreux rapports font état d'une désaffectation à l'égard des matières scientifiques dans l'enseignement supérieur, notamment en France (Giret et al., 2006). Outre la question en termes d'offre de diplômés en S\&I (Sciences et Ingénieries) se pose celle du choix de carrière de ces jeunes diplômés (Lavoie et Finnie, 1998). Aux USA, Lowell et Salzman (2007) ne constatent pas de pénurie en termes d'offre de diplômés ; en revanche, il semblerait que ces jeunes diplômés puissent être attirés par d'autres types de carrières plus attractives. Selon Murphy et al. (1991), la concentration d'individus les "plus talentueux" au sein des métiers de la finance ou du juridique peut être une des sources d'une moindre croissance de productivité pour un pays.

Dès lors, il est pertinent de comprendre l'insertion des diplômés en sciences au sein des activités de R\&D privée. Une difficulté réside dans l'incertitude sur la productivité de l'individu, que nous pouvons supposer plus importante que dans le secteur hors recherche. Lors de l'embauche, il est difficile pour l'employeur d'évaluer les connaissances de l'individu et ses capacités à résoudre des problèmes ou plus généralement à innover (Zenger, Lazzarini, 2004). Rares sont les études sur la productivité du chercheur industriel. Nous pouvons nous interroger sur les facteurs qui déterminent l'accès à la R\&D. En effet, lors du recrutement, l'employeur se base-t-il uniquement sur le capital humain de l'individu (signal "formel ») ou utilise-t-il d'autres sources d'informations telles que les réseaux 
de l'individu (signal «informel ") ? Ces facteurs différent-ils selon le type de diplôme ? Comment le diplôme scientifique est-il valorisé au sein des activités de R\&D ?

L'analyse de l'accès à la R\&D est confrontée à une difficulté : les diplômés travaillant dans le secteur recherche peuvent présenter une motivation différente, telle qu'une préférence pour la recherche, par rapport aux diplômés exerçant dans un autre secteur d'activité. Or, ce type de motivation est inobservable, ce qui risque d'entraîner des biais de sélection. En effet, ces caractéristiques non observables sont susceptibles d'affecter à la fois le fait de travailler dans la $R \& D$ et le salaire des jeunes diplômés. Afin de corriger ces biais, nous utilisons un modèle de régression endogène (" endogenous switching regression») ou encore appelé "Mover/stayer ". Cette méthode estime simultanément les fonctions de gains et l'équation de sélection par la méthode du maximum de vraisemblance à information complète ( $c f$. encadré 1). Les données utilisées sont issues de l'enquête 2007 sur la " Génération 2004 ", réalisée par le Céreq. Cette enquête concerne environ 33000 individus interrogés en 2007 sur leur insertion professionnelle, soit trois ans après leur sortie du système éducatif. Nous nous intéressons ici uniquement aux diplômés possédant au moins un bac +5 dans une discipline scientifique, et salariés du secteur privé, ce qui représente environ 1500 individus dans l'échantillon. Dans un premier temps, les déterminants de l'accès aux emplois de $\mathrm{R} \& \mathrm{D}$ ( $c f$. encadré 2 ) privée sont analysés pour l'ensemble des jeunes diplômés en sciences. Les modèles sont également estimés séparément pour les docteurs et les diplômés d'écoles d'ingénieurs. Il s’agit de déterminer s'il existe une différenciation quant à la sélection dans les activités de R\&D entre ces deux diplômes. Dans un second temps, la rémunération des jeunes diplômés est analysée dans le secteur recherche et hors recherche. Cette analyse permettra, en particulier, de comparer le rendement salarial des différents diplômes dans les deux types d'activités. Enfin, à partir des paramètres des modèles estimés, les salaires conditionnels et non conditionnels sont prédits ; cela permet de mesurer l'écart salarial entre les activités de R\&D et les autres activités pour chaque diplôme.

\section{Les déterminants de l'accès aux emplois de R\&D}

Dans le secteur privé, lors du recrutement, il est malaisé pour l'employeur de déterminer la productivité du jeune chercheur. En effet, les tâches de l'ingénieur étant principalement cognitives, l'employeur peut difficilement évaluer les capacités du jeune scientifique à innover (Zenger et Lazzarini, 2004). Dans le secteur académique, la productivité du jeune chercheur est généralement estimée par le nombre de publications au cours de son doctorat (Levin et Stephan, 1991). Des études montrent qu'en France, le nombre de publications ne semble pas être un critère de recrutement dans le secteur privé (Giret $e t$ al., 2007 ; Mangematin, 2000). Dans cette partie, nous analysons les facteurs sur lesquels l'employeur peut s'appuyer. 


\section{Encadré 1}

\section{Méthodologie : Modèle de régression endogène}

Des travaux (Giret, Perret, Recotillet, 2003 et Lassibille, 2001) montrent l'existence de caractéristiques non observables pouvant entraîner des biais de sélection en affectant à la fois le fait de travailler dans la recherche et le salaire. Dans ce papier, nous utilisons le modèle Mover/stayer, encore appelé " endogenousswitchingregression », qui a l'avantage d'estimer simultanément les fonctions de gains et l'équation de sélection par la méthode du maximum de vraisemblance à information complète, permettant d'avoir des erreurs standards plus robustes (Loksin, Zurab, 2004).

Le modèle prend la forme suivante:

(2.1.) $\ln \left(w_{1 i}\right)=$ cste $+\beta_{1} X_{1 i}+\varepsilon_{1 i}$ si $I_{1}^{*}>I_{0}^{*}$

(2.2.) $\ln \left(w_{0 i}\right)=$ cste $+\beta_{0} X_{0 i}+\varepsilon_{0 i}$ si $I_{1}^{*}<I_{0}^{*}$

(2.3. $) \mathrm{I}_{\mathrm{i}}^{*}=\mathrm{Z}_{\mathrm{i}} \gamma+\mathrm{u}_{\mathrm{i}}$

Les équations 2.1 et 2.2 correspondent aux fonctions de gains respectivement dans le secteur de la recherche et le secteur hors recherche $w_{1 i}$ et $w_{0 i}$ sont respectivement le salaire dans la recherche et hors recherche $X_{1 i}$ et $X_{0 i}$ sont des variables de capital humain et de capital social permettant d'expliquer le salaire. L'équation 2.3 correspond à l'équation de sélection, où $I_{1}^{*}$ est une variable latente déterminant la décision du diplômé face aux choix de travailler dans le secteur de la recherche ou hors recherche. Puisque $I_{1}^{*}$ ne peut être observée par le chercheur, elle correspond à une variable dichotomique où $l=1$ si les diplômés choisissent de travailler dans la recherche et 0 s'ils choisissent de travailler dans d'autres activités. Soit :

$Z_{i}$ est composé de variables individuelles et de variables instrumentales, ces dernières affectant le choix de travailler dans un des deux secteurs mais n'ayant pas d'impact direct sur le salaire. $u_{i}$ et $\varepsilon_{1 i}, \varepsilon_{2 i}$ sont les termes d'erreur respectivement de l'équation de sélection et des fonctions de gains. Le modèle estime également les coefficients de corrélation entre $\varepsilon_{1 i}$ et $\mathbf{u}_{i}$ et $\varepsilon_{2 i}$ et $\mathbf{u}_{i}$, soient $p_{1}$ et $p_{0}$. Une attention particulière doit être portée sur ces coefficients. En effet, si $p_{1}$ ou $p_{0}$ est statistiquement différent de zéro, le terme d'erreur de l'équation de sélection est corrélé avec les termes d'erreur des équations de salaire. En d'autres termes, la sélection au sein d'un secteur particulier est endogène au salaire. Des caractéristiques ou des préférences non observées influençant le fait d'être dans la recherche ou pas ont probablement une influence sur le salaire des diplômés une fois qu'ils sont employés (Loksin et Zurab, 2004). Par ailleurs, l'existence de biais d'auto-sélection justifie l'adoption d'un tel modèle par rapport au modèle classique des MCO (moindres carrés ordinaires) qui générerait des estimations biaisées.

En effet, après l'estimation des modèles, il est possible de calculer les salaires contrefactuels pour chaque cas. Nous calculons $y c_{1 \_i i}\left(y c_{0 \_0 i}\right)$ correspondant aux salaires prédits des diplômés actuellement dans la R\&D (resp. hors recherche) conditionnellement au fait qu'ils travaillent dans la R\&D (resp. dans des activités hors recherche). Puis, les salaires contrefactuels $y c_{0_{1} i i}$ et $y c_{1 \_i i}$ correspondant aux salaires des diplômés actuellement dans la R\&D (resp. hors R\&D) s'ills se trouvaient dans des activités hors recherche (resp. R\&D).

A partir de ces salaires prédits, deux indicateurs peuvent être construits :

$$
\begin{aligned}
& \Pi_{1}=y c_{1 \_1 i}-y c_{0 \_1} i \\
& \Pi_{0}=y c_{1 \_0 i}-y c_{0 \_0 i}
\end{aligned}
$$


$\Pi_{1}$ mesure la différence de salaire entre être employé dans le secteur R\&D et être employé dans le secteur hors recherche pour les diplômés se trouvant actuellement dans la recherche. Si $\Pi_{1}$ est positif (resp. négatif), le secteur R\&D est plus (resp. moins) rémunérateur que le secteur hors recherche pour les diplômés actuellement employés dans la R\&D.

$\Pi_{0}$ mesure la différence de salaire entre être employé dans le secteur R\&D et être employé dans le secteur hors recherche pour les diplômés se trouvant actuellement dans le secteur hors recherche. Dans ce cas, si $\Pi_{0}$ est positif (négatif), le secteur R\&D est plus (moins) rémunérateur que le secteur hors recherche pour les diplômés actuellement employés dans le secteur hors recherche.

Notes : Les modèles sont estimés sous Stata.

Dans un premier temps, le modèle comprend l'ensemble des diplômés à au moins Bac +5 (master en sciences, écoles d'ingénieurs et docteurs). Dans un second temps, les modèles sont estimés séparément pour les diplômés d'écoles d'ingénieurs et les docteurs.

La commande movestay est utilisée pour l'estimation des modèles.

\section{Encadré 2}

\section{Définition des activités de R\&D (*)}

Les diplômés considérés comme travaillant dans la R\&D privée sont ceux ayant les PCS (professions et catégories sociales) suivantes:

383A : Ingénieurs et cadres d'étude recherche et développement en électricité électronique.

384A : Ingénieurs et cadres d'étude recherche et développement en mécanique et travail des métaux.

385A : Ingénieurs et cadres d'étude recherche et développement des industries de transformation (agroalimentaire, chimie, métallurgie, matériaux lourds).

386A : Ingénieurs et cadres d'étude recherche et développement des autres industries.

474B: Techniciens de recherche-développement et des méthodes de fabrication en construction mécanique et travail des métaux.

475A : Techniciens de recherche-développement et des méthodes de production des industries de transformation.

479A : Techniciens des laboratoires de recherche publique ou de l'enseignement.

625B : Ouvriers qualifiés et agents qualifiés de laboratoire : agroalimentaire, chimie, biologie, pharmacie.

$\left(^{*}\right)$ : Pour la définition de la R\&D privée, on utilise la typologie d'Audric Lerenard et Topiol (1999). 


\subsection{Recrutement : I'avantage aux diplômés d'écoles d'ingénieurs}

Tout d'abord, l'employeur peut s'appuyer sur le niveau de capital humain du jeune diplômé. Le principal débouché des docteurs est la recherche publique. Néanmoins, ces dernières années, il semble que le secteur privé devient de plus en plus attractif pour ces derniers. D'ailleurs, l'un des objectifs de la formation doctorale initiée par les pouvoirs publics est de favoriser l'accès des jeunes docteurs aux emplois de R\&D dans le secteur privé (Giret et al., 2007). La politique actuelle, qui vise à rapprocher le secteur académique des laboratoires de $\mathrm{R} \& \mathrm{D}$ privée, nous incite à supposer que le doctorat est privilégié par rapport aux autres diplômes dans le secteur R\&D.

La littérature énumère les nombreux avantages liés à l'embauche de docteurs dans les laboratoires de R\&D. Les docteurs sont, par exemple, plus susceptibles de renforcer les " capacités d'absorption " des entreprises en matière de connaissance scientifique par rapport aux autres diplômés (Giret et al, 2007). En effet, leur formation leur permet plus facilement de suivre les nouvelles découvertes scientifiques académiques et de les appliquer à la R\&D industrielle. Pour autant, les entreprises préferrent largement les diplômés d'écoles d'ingénieurs (Observatoire de l'emploi scientifique, 2009). On reproche aux docteurs leur méconnaissance du monde de l'entreprise, contrairement aux diplômés des écoles d'ingénieurs qui effectuent un stage long en entreprise (Duhautois et Maublanc, 2005, 2006). D'autre part, lors de leur carrière, les chercheurs en entreprise sont souvent amenés à se diriger vers d'autres fonctions, managériales notamment (Biddle et Roberts, 1994) ou vers des fonctions de production et de marketing (Duhautois et Maublanc, 2005). En effet, Beltramo, Paul et Perret(2000) soulignent que la R\&D peut jouer un rôle de "pépinière " au sein des entreprises. Ainsi, les entreprises privilégient l'embauche de diplômés d'écoles d'ingénieurs. Elles considèrent qu'ils ont suivi une formation plus générale et ont acquis les compétences managériales nécessaires pour se diriger vers d'autres fonctions, contrairement aux docteurs. Ce résultat doit cependant être nuancé, les docteurs ayant un cursus antérieur en écoles d'ingénieurs ont plus de chances d'accéder aux fonctions de R\&D privée. Ils représentent $23 \%$ des docteurs-chercheurs dans le privé.

\subsection{L'expérience comme complément au diplôme}

Un autre aspect du capital humain à prendre en considération est l'expérience professionnelle antérieure en entreprise du jeune diplômé, notamment pour les détenteurs d'un doctorat. L'expérience du monde industriel permet d'accroître le capital humain scientifique du jeune diplômé, d'acquérir des compétences particulières (Lam, 2001). L'expérience peut être également perçue par l'employeur comme un signal d'une adaptation du docteur au monde de l'entreprise. Les travaux portant sur les docteurs semblent confirmer cette hypothèse. En effet, il semble que les docteurs accédant au secteur privé sont ceux qui ont eu une expérience avec le monde industriel lors de leur doctorat. Cette expérience a été acquise grâce au financement dont ils ont bénéficié pendant leur doctorat, comme 
les conventions CIFRE $^{1}$, qui permettent aux docteurs d'effectuer leur thèse à l'université ainsi qu'en entreprise, ou encore en participant à des contrats de recherche avec le secteur privé (Mangematin, 2000 ; Giret et al., 2007).

\subsection{L'établissement de formation comme signal de qualité}

L'établissement de formation peut également être utilisé par les employeurs comme intermédiaire lors de l'embauche. Par exemple, au Japon, Rebick (2000) montre que les liens tissés entre les universités et les entreprises permettent aux employeurs de réduire les coûts de recrutement et d'améliorer la sélection des employés. Il souligne, dans le cas des sciences, que les professeurs peuvent, par exemple, jouer un rôle d'intermédiaire en plaçant les meilleurs étudiants dans les entreprises avec lesquelles ils entretiennent des liens. En outre, ces liens permettent aux diplômés de connaître les possibilités d'embauche dans ces entreprises. Lam (2001) souligne que les entreprises tendent à entretenir des liens étroits avec les universités afin d'avoir accès aux meilleurs étudiants et pouvoir influencer la formation au sein des entreprises, ce que l'auteur nomme marché interne étendu. Elle souligne que ces réseaux offrent au recruteur plus d'informations sur la qualité des compétences que le diplôme possédé par le candidat. Enfin, les entreprises peuvent également se référer au prestige de l'établissement de formation des diplômés, particulièrement dans le cas des écoles d'ingénieurs. En effet, en France, le prestige des différentes écoles d'ingénieurs est bien connu des entreprises, notamment grâce aux classements annuels des écoles publiés dans différents journaux et magazines, malgré les limites inhérentes à ces classements ( $c f$. dans ce numéro, l'article de Bourdon, Giret et Goudard, sur le classement des universités). Les écoles d'ingénieurs présentent aussi une forte hétérogénéité en termes de poids accordé à l'enseignement de la recherche.

\subsection{Les réseaux pour sélectionner les individus}

Par ailleurs, lorsque l'incertitude sur la productivité est importante, le niveau de capital humain peut s’avérer insuffisant. Les employeurs peuvent être amenés à utiliser un référé, un signal « informel », selon l'expression de Saloner (1985), lors du recrutement des jeunes chercheurs. De nombreuses études ont analysé le rôle des réseaux sociaux lors du recrutement. Granovetter (1995) montre le rôle informationnel des réseaux sociaux sur le marché du travail. Selon lui, les réseaux permettent aux employeurs et employés d'obtenir une information plus fiable et de meilleure qualité lors du recrutement. Montgomery (1991) et Saloner (1985) soulignent également que les réseaux sociaux permettent de réduire

1. CIFRE : Convention Industrielle de Formation par la Recherche. L'objectif est de favoriser le développement de la recherche partenariale publique-privée et de placer les doctorants dans des conditions d'emploi. Elle repose sur l'association de quatre acteurs : l'entreprise qui recrute en CDI ou CDD un diplômé de niveau master, le laboratoire de recherche académique qui encadre les travaux du salarié-doctorant, le doctorant et l'ANRT qui contracte avec l'entreprise une convention sur la base de laquelle une subvention est versée à l'entreprise. 
l'asymétrie d'information entre les employeurs et les employés. L'ensemble de ces études conclut que les individus utilisant les réseaux sociaux ont une plus grande probabilité de trouver un emploi mieux payé. Cependant, Franzen et Hangarten (2006) estiment que les réseaux sociaux des diplômés ne permettent pas à ceux-ci d'obtenir des emplois mieux rémunérés mais un meilleur appariement entre le niveau d'éducation et l'emploi. Dans le cas de la R\&D, Simon et Warner (1992) montrent, par exemple, que les employeurs utilisent les réseaux d'anciens élèves lors de l'embauche des jeunes chercheurs afin de réduire l'incertitude sur la productivité du futur employé. Dans une étude plus récente, Mangematin (2000) souligne l'importance du réseau social pour les docteurs dans l'accès au secteur privé.

\subsection{Le salaire d'embauche comme moyen de gérer les entrées de carrière}

Pour faire face à l'asymétrie d'information sur la productivité du jeune chercheur, l'employeur peut également agir sur le salaire d'embauche. En effet, selon Weiss (1980), lorsque l'incertitude sur les capacités des individus est importante, l'employeur peut décider d'offrir des salaires d'embauche plus élevés afin d'attirer les individus les plus talentueux. Cependant, l'incertitude sur la productivité du jeune chercheur peut avoir l'effet inverse sur le salaire d'embauche. En effet, Mishagina (2008) montre que, puisqu'en début de carrière, l'information sur la productivité du jeune chercheur n'est pas connue, l'employeur peut décider d'offrir des salaires inférieurs aux individus travaillant dans le secteur recherche par rapport à ceux exerçant d'autres activités. Lorsque l'information sur les capacités de l'individu à faire de la recherche est révélée, les chercheurs les moins productifs vont quitter la recherche pour d'autres activités alors que les plus productifs resteront dans la recherche et connaîtront une croissance de salaire plus élevée. Selon cette théorie, un salaire d'embauche moins important provient d'un "effet productivité" . Moen (2005) apporte cependant une justification différente. Selon cet auteur, un salaire inférieur pourrait s'expliquer par un "effet apprentissage ", les fonctions de R\&D étant perçues comme un investissement en capital humain de la part des jeunes diplômés, qu'ils pourront ensuite faire valoriser.

Certaines théories suggèrent que le salaire d'embauche peut également être utilisé afin d'induire une auto-sélection des candidats. Heyes (2004) montre ainsi que, dans le cas des métiers à forte vocation ${ }^{2}$, proposer des salaires d'embauche trop élevés risque d'attirer les candidats les moins motivés. Delfgaauw et Dur (2007) reprennent cet argument dans un contexte plus général. Ils supposent une forte hétérogénéité des candidats quant à leur motivation intrinsèque. Dès lors, offrir des salaires élevés peut amener les individus les moins motivés à se présenter et diminue donc la " qualité " des candidats. Cette théorie peut s'appliquer au cas des chercheurs dans la mesure où il a été montré qu'ils pouvaient présenter une motivation intrinsèque importante, un certain "goût pour la recherche " (Stern, 2004).

2. Dans son étude, il prend l'exemple des infirmières. 


\section{Présentation des données}

Les données sont issues de l'enquête 2007 sur la " Génération 2004 », réalisée par le Céreq. Elle concerne environ 33000 individus, interrogés en 2007 sur leur insertion professionnelle, soit 3 ans après leur sortie du système éducatif. Les données de l'enquête permettent de recueillir les informations nécessaires sur l'individu ainsi que sur l'emploi occupé 3 ans après l'obtention du diplôme. Dans l'échantillon, 1513 diplômés possèdent au moins un bac + 5 dans une discipline scientifique et sont salariés dans le secteur privé. 31,5\% travaillent en R\&D contre 68,5\% dans le secteur hors recherche.

\subsection{Variables individuelles et d'entreprise}

Les variables individuelles correspondent au sexe, aux diplômes et à la discipline du diplôme. Comme nous l'avons vu, l'expérience professionnelle en entreprise des docteurs semble déterminante dans l'accès au secteur privé. Nous construisons donc une variable d'interaction prenant en compte le suivi ou non d'un stage en entreprise lors de ces études. Dans le modèle relatif aux ingénieurs, on prend en compte le fait que l'individu a obtenu ou non un autre diplôme que celui d'ingénieur. Le modèle concernant les docteurs intègre le fait que le docteur a effectué ou non un stage en entreprise ${ }^{3}$; lors de ses études, le type de financement (CIFRE) dont il a bénéficié, ainsi que le projet professionnel lors du doctorat (recherche publique/autre projet).

La prise en compte du parcours professionnel du jeune diplômé est également importante. Pour cela, nous utilisons le nombre de mois en emploi ainsi que le nombre de périodes de chômage. Enfin, sont aussi considérées les caractéristiques de l'entreprise. Les politiques de rémunération et de recrutement au sein des activités de R\&D peuvent, en effet, différer selon la taille, l'activité et la région d'implantation de l'entreprise.

\subsection{Variables d'accès à l'emploi}

Dans cet article, nous nous centrons sur le moyen de recrutement utilisé pour les activités de R\&D. Cette variable est tirée de l'item de l'enquête suivant : " Comment avez-vous su qu'il y avait une embauche possible dans cette entreprise? ». Les réponses possibles sont : " par votre établissement de formation ", " par une de vos relations » et " autres".

Les relations représentent un mode de recrutement fréquent pour les diplômés à bac +5 . En effet, en moyenne, $27 \%$ des diplômés ont été informés d'une offre d'emploi par cet intermédiaire. Les docteurs semblent avoir plus recours à leurs relations que les autres

3. Pour le stage en entreprise, ne sont pas pris en compte les docteurs ayant bénéficié d'un financement CIFRE lors de leur doctorat. 
Tableau 1

Comment avez-vous su qu'il y avait une embauche possible dans cette entreprise?

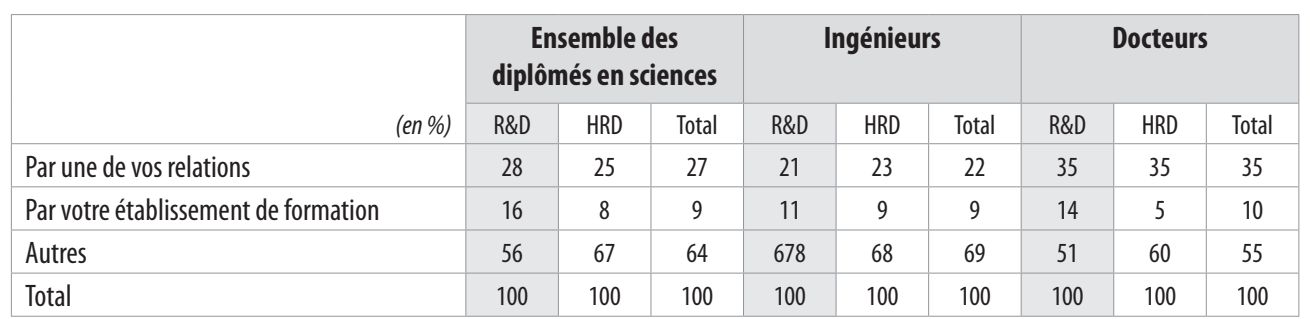

Source : auteur, à partir de l'enquête Céreq 2007 sur la Génération 2004.

Note de lecture : $28 \%$ des diplômés en sciences ont su qu'il y avait une embauche possible par une de leurs relations.

diplômés (35\% contre $27 \%$ ). Mangematin (2000) souligne que les docteurs utilisent fréquemment leurs relations pour trouver un emploi dans le secteur privé. Le tableau 1 montre également qu'une part plus importante des diplômés travaillant dans le secteur recherche a utilisé son établissement de formation par rapport aux diplômés employés dans le secteur hors recherche.

\subsection{Variables écoles d'ingénieurs}

Dans le modèle relatif aux ingénieurs, le prestige de l'école est également pris en compte ${ }^{4}$. En outre, à partir du classement effectué par la revue "Industrie et Technologies ", les écoles d'ingénieurs dans l'échantillon peuvent être classées à l'aune du montant du chiffres d'affaires (prestations techniques, contrats de R\&D, programmes européens) qu'elles ont réalisé avec les entreprises en 2006. Les vingt premières écoles ont été regroupées en une variable nommée «Ecole R\&D ». Ces écoles réalisent en moyenne 3,15 millions d'euros de CA avec les entreprises. Nous avons construit cette variable car nous pouvons supposer que ces écoles entretiennent des liens étroits avec les laboratoires de R\&D des entreprises. 12,6 \% des ingénieurs travaillant en $\mathrm{R} \& \mathrm{D}$ sont diplômés d'une école prestigieuse contre 9,4\% des ingénieurs exerçant des activités hors recherche. D’autre part, 22,5\% des ingénieurs qui exercent des activités de R\&D sont issus d'une « Ecole R\&D » contre 15,1 \% des diplômés travaillant dans le secteur hors recherche.

\subsection{Variables instrumentales}

Dans le modèle, il s'agit d'introduire une variable instrumentale au sein de l'équation de sélection. Il s'agit d'une variable permettant de prédire l'accès à la recherche du jeune diplômé mais n'ayant pas d'effet direct sur le salaire. La variable choisie concerne le projet

4. Les écoles prestigieuses sont les écoles classées dans les dix premières dans différents magazines (Le point, L'étudiant...). 
professionnel des diplômés l'année du bac. En effet, la littérature (Fox et Stephan, 2001) montre qu'un intérêt pour la science, dès le plus jeune âge, est un prédicteur d'une future carrière scientifique. Cette variable peut être considérée comme une variable proxy d'un goût pour la science de la part des individus. Elle est construite à partir des items concernant le métier et le domaine souhaités par le diplômé au moment du bac. Cette variable présente six modalités : lors de l'année du bac, le diplômé souhaite travailler dans le domaine de la recherche, de la science, de l'informatique, en tant qu'ingénieur, dans un autre domaine et, enfin, n'a pas de projet.

Globalement, une part plus importante de diplômés dans le secteur hors recherche n’avait aucun projet professionnel lors de l'année du bac, par rapport aux diplômés actuellement dans le secteur recherche (21,4\% contre 15,7\%). Les salariés du secteur recherche se projetaient plus fréquemment travaillant dans le domaine de la recherche, de la science ou en tant qu'ingénieur. En revanche, peu d'entre eux souhaitaient exercer dans le domaine de l'informatique (3,3\% contre 14,9\% pour les diplômés actuellement dans d'autres activités). Enfin, pour les estimations des docteurs, s'ajoute une variable instrumentale ayant trait au fait que le docteur a effectué ou non un stage post-doctorat. En effet, Recotillet (2007) montre que le stage post-doctorat peut jouer le rôle de signal dans le début de carrière des docteurs mais n'apporte pas de gain salarial dans le secteur privé. Dans l'échantillon, $40,9 \%$ des docteurs dans le secteur recherche ont effectué un stage post-doctorat contre $24,8 \%$ dans le secteur hors recherche.

\section{Résultats}

Dans cette partie, nous présentons, tout d'abord, les déterminants, pour les jeunes diplômés en sciences, de l'accès aux fonctions de R\&D privée. Puis, nous comparons les rendements des différentes caractéristiques des diplômés au sein des activités de R\&D et hors des activités de R\&D. Enfin, nous présentons les salaires conditionnels et contrefactuels. Il s'agit de déterminer le salaire à l'entrée dans la recherche, une fois les biais de sélection pris en considération. L’ensemble des résultats sont présentés en annexe de la version électronique du présent document dans les tableaux $2 \mathrm{~A}, 2 \mathrm{~B}$ et $2 \mathrm{C}$ (http://www. cairn.info/revue-formation-emploi-2011).

\subsection{L'effet discipline et l'impact de l'expérience professionnelle}

Les diplômés titulaires d'un doctorat et ceux issus d'une école d'ingénieurs ont une plus forte probabilité d'accéder à la $\mathrm{R} \& \mathrm{D}$ que les diplômés d'un master en sciences. La probabilité est particulièrement élevée pour les docteurs-ingénieurs et les docteurs-universitaires ${ }^{5}$ ayant effectué un stage en entreprise lors de leurs études. Il apparait également

5. Nous appelons docteurs-universitaires, les docteurs avec un cursus universitaire et docteurs-ingénieurs, les 
un « effet discipline ». En effet, être diplômé en sciences de l'ingénierie ou en agronomie décroît la probabilité d'accéder à la R\&D par rapport aux diplômés en technologie.

Les résultats révèlent aussi une différenciation des facteurs d'accès à la $R \& D$ entre les docteurs et les diplômés d'écoles d'ingénieurs. En effet, pour les docteurs, le mode de financement apparaît déterminant dans l'accès à la $\mathrm{R} \& \mathrm{D}$ privée. Le fait d'avoir bénéficié d'un financement CIFRE lors du doctorat accroît fortement la probabilité d'accéder à la R\&D privée par rapport à un autre type de financement. Le cursus antérieur du docteur (écoles d'ingénieurs vs. universitaire) ne semble pas avoir d'impact sur la probabilité d'accès à la R\&D. En revanche, avoir effectué un stage en entreprise lors de ses études est fortement positif et significatif. Au vu de ces résultats, l'expérience professionnelle en entreprise et dans la recherche privée acquise avant et lors du doctorat (CIFRE, stage) semble être le facteur de sélection déterminant l'accès à la R\&D privée.

Pour les diplômés d'écoles d'ingénieurs, il semble que l'école dont ils sont diplômés intervienne comme un effet signal important pour accéder aux activités de R\&D. Ainsi, le fait que le diplômé soit issu d'une " école $\mathrm{R} \& \mathrm{D}$ " accroît fortement ses chances de devenir chercheur (significatif à $5 \%$ ). Dès lors, les ingénieurs issus d'une école ayant tissé de nombreux liens avec les laboratoires de R\&D des entreprises semblent avoir plus de probabilité d'occuper des emplois dans la recherche.

Quant au moyen par lequel les diplômés ont pris connaissance d'une possible embauche, les relations personnelles apparaissent non significatives dans l'ensemble des modèles. Le fait d'avoir été informé d'une possible embauche par l'établissement de formation accroît significativement (au seuil de $5 \%$ ) les chances d'appartenir au secteur recherche pour l'ensemble des diplômés en sciences ainsi que pour les diplômés d'écoles d'ingénieurs. Avoir été informé par le biais de l'établissement de formation peut correspondre à différentes situations. En effet, les diplômés ont pu avoir connaissance de l'offre d'emploi par un de leurs professeurs ou par une annonce déposée dans leur établissement ou encore par les réseaux d'anciens élèves.

La variable instrumentale utilisée dans les modèles, soit le projet souhaité l'année du bac, est également significative. En effet, les diplômés souhaitant travailler dans la recherche ou en tant qu'ingénieur ont de plus fortes chances de travailler dans le secteur R\&D alors que ceux se projetant dans le domaine de l'informatique ont une plus faible probabilité de s'y trouver par rapport aux diplômés sans projet professionnel ${ }^{6}$. Pour les docteurs, le fait d'avoir effectué un stage post-doctorat accroît également fortement les chances de devenir chercheur. Notons que l'introduction des variables concernant l'entreprise modifie peu les résultats.

docteurs avec un cursus antérieur en école d'ingénieurs.

6. On ne peut toutefois pas écarter la probabilité de rationalisation a posteriori, les jeunes travaillant dans la R\&D déclarant plus volontiers souhaiter y travailler dès leur bas âge, tandis que ceux n'y travaillant pas déclarent moins volontiers ne pas souhaiter y travailler au moment du baccalauréat. 


\subsection{La rémunération dans la $R \& D$ : fonctions de gains}

Le diplôme a un effet positif significatif sur le revenu, mais le rendement des diplômes diffère selon les deux types d'activités. En effet, dans le secteur de la R\&D, être titulaire d'un doctorat engendre un gain salarial significatif par rapport aux diplômés du $3^{\text {ème }}$ cycle (master sciences et écoles d'ingénieurs). Le rendement salarial est particulièrement important pour les docteurs-ingénieurs et les docteurs-universitaires ayant effectué un stage en entreprise, soit respectivement environ $+38 \%$ et $+34 \%$ par rapport aux diplômés d'un master sciences. Dans le domaine hors recherche, le rendement positif du doctorat est beaucoup plus faible. Être docteur avec un cursus universitaire et ne pas avoir effectué de stage en entreprise engendre un gain salarial significatif de $9 \%$ par rapport aux diplômés d'un master sciences, alors que le gain salarial est de $29 \%$ dans le secteur recherche. Les docteurs-ingénieurs et les docteurs-universitaires ayant effectué un stage en entreprise ont un rendement salarial significativement positif par rapport aux diplômés en master sciences. Cependant, celui-ci est en moyenne deux fois moins important que dans le domaine de la R\&D. En revanche, seuls les docteurs avec un cursus en école d'ingénieurs bénéficient d'un gain salarial significatif par rapport aux diplômés d'écoles d'ingénieurs. Le doctorat apparait donc beaucoup plus valorisé dans le secteur de la R\&D que dans les autres activités au sein des entreprises.

La discipline du diplôme a un impact similaire sur le salaire dans les deux types d'activités. Par rapport à la discipline technologie, les disciplines agronomie et autres sciences de l'ingénierie semblent avoir un impact négatif sur le salaire, que ce soit dans le secteur recherche ou hors recherche.

S’agissant plus particulièrement des docteurs, le financement dont ils ont bénéficié lors de leur doctorat a un impact salarial semblable dans les deux types de secteurs. En effet, le financement CIFRE est valorisé à la fois dans le secteur recherche (18,5\%) et le secteur hors recherche $(21 \%)$. Le cursus antérieur en école d'ingénieurs entraîne un gain salarial (significatif à 10 \%) uniquement dans le secteur hors recherche. À cet égard, une étude récente de Riedinger et Zaiem (2011) montre quau sein des activités de R\&D, les docteurs-ingénieurs ne sont pas plus productifs (mesurés par le nombre de brevets déposés) que les docteurs universitaires. Ce résultat peut expliquer le fait que les docteursingénieurs ne bénéficient pas d'un rendement salarial supérieur par rapport aux autres docteurs au sein des activités de R\&D. En revanche, le stage ${ }^{7}$ présente un coefficient significatif positif dans les deux secteurs. Un docteur ayant effectué un stage en entreprise durant ses études percevra un salaire supérieur de $9 \%$ dans le secteur R\&D et de $14 \%$ dans le secteur hors recherche.

7. Les docteurs ayant bénéficié d'un financement CIFRE ne sont pas comptabilisés ici comme ayant suivi un stage en entreprise. 
Pour les diplômés d'écoles d'ingénieurs, nous retrouvons le rôle de "signal » des écoles d'ingénieurs. Les ingénieurs issus d'une école prestigieuse perçoivent des revenus supérieurs à ceux des ingénieurs issus des autres écoles. En effet, être diplômé d'une école prestigieuse entraîne un gain de 14 \% dans le secteur recherche et de 11 \% dans le secteur hors recherche. En revanche, être diplômé d'une «école $\mathrm{R} \& \mathrm{D}$ » engendre un gain positif (8\%, significatif à $5 \%)$ uniquement dans le secteur recherche.

Les résultats des différents modèles révèlent également que certaines caractéristiques des jeunes diplômés engendrent un rendement différent dans le secteur $R \& D$ et le secteur hors recherche. Tout d'abord, l'écart salarial entre les hommes et les femmes est beaucoup plus faible dans le secteur recherche que dans le secteur hors recherche. En effet, pour l'ensemble des diplômés en sciences, l'écart de salaire entre les hommes et les femmes est de $7 \%$ au sein des activités de R\&D alors qu'il est de $10 \%$ au sein des activités hors recherche. Aux USA, Graham et Smith (2005) notent également que la discrimination salariale envers les femmes est bien moindre dans le domaine de la recherche que dans les autres activités. Soulignons que nous nous situons en début de carrière, l'écart de rémunération entre les hommes et les femmes peut apparaître par la suite. L'expérience professionnelle du jeune diplômé apparaît moins valorisée dans le secteur recherche que dans le secteur hors recherche. Le rendement de l'expérience professionnelle est de $0,4 \%{ }^{8}$ (significatif à $5 \%$ ) dans le secteur recherche contre $0,7 \%$ dans le secteur hors recherche.

Enfin, s'agissant des variables entreprises, nous retrouvons les résultats classiques. Le fait d'être employé dans une entreprise de taille importante et de travailler en région parisienne entraine un gain salarial positif, que ce soit dans le secteur $R \& D$ ou dans le secteur hors recherche.

\subsection{Un effet de préférence pour la recherche?}

Il semble que le secteur hors recherche tende à offrir des salaires supérieurs à ceux du secteur R\&D aux docteurs actuellement en R\&D et aux docteurs actuellement en hors recherche. Les estimations des docteurs révèlent que les indicateurs $\pi_{1}$ et $\pi_{0}$ sont significativement négatifs, que ce soit dans les modèles avec variables individuelles ou variables entreprises. Ainsi, dans le cas des docteurs, les activités de R\&D sont moins rémunératrices que les activités hors recherche. Ce résultat peut donc suggérer une "préférence " des docteurs pour la recherche; en effet, les docteurs actuellement employés dans le secteur R\&D pourraient percevoir des salaires supérieurs ( $40 \%)$ s'ils exerçaient des activités hors recherche. Ce résultat demanderait à être approfondi par spécialité de formation ou encore par secteur d'activité. Par ailleurs, nous nous situons ici en début de carrière (trois

8. L'expérience professionnelle est mesurée par le nombre de mois passés en emploi. 


\section{Tableau 2}

\section{Écart salarial entre les deux types d'activités}

\begin{tabular}{|c|c|c|c|c|c|}
\hline & \multirow[b]{2}{*}{$\begin{array}{r}\text { Modèle } \\
\text { par les MCO } \\
\text { Coefficient } \\
\text { de la } \\
\text { variable R\&D }\end{array}$} & \multicolumn{2}{|c|}{$\begin{array}{c}\text { Indicateurs } \\
\text { Modèle avec variables } \\
\text { individuelles }\end{array}$} & \multicolumn{2}{|c|}{$\begin{array}{c}\text { Indicateurs } \\
\text { Modèle avec variables } \\
\text { entreprise }\end{array}$} \\
\hline & & $\begin{array}{l}\text { Différence de salaire } \\
\text { entre emploi R\&D } \\
\text { et emploi non } R \& D \\
\text { au sein du secteur } \\
\text { recherche } \\
\left(\pi_{1}\right)\end{array}$ & $\begin{array}{l}\text { Différence de salaire } \\
\text { entre emploi } R \& D \text { et } \\
\text { emploi non } R \& D \text { au } \\
\text { sein du secteur hors } \\
\text { recherche } \\
\left(\pi_{0}\right)\end{array}$ & $\begin{array}{l}\text { Différence de salaire } \\
\text { entre emploi } R \& D \\
\text { et emploi non } R \& D \\
\text { au sein du secteur } \\
\text { recherche } \\
\left(\pi_{1}\right)\end{array}$ & $\begin{array}{l}\text { Différence de salaire } \\
\text { entre emploi } R \& D \text { et } \\
\text { emploi non } R \& D \text { au } \\
\text { sein du secteur hors } \\
\text { recherche } \\
\left(\pi_{0}\right)\end{array}$ \\
\hline Ensemble des diplômés & 0,01 & $0,02^{* * *}$ & $-0,40^{* * *}$ & $-0,01^{* * *}$ & $-0,41^{* * *}$ \\
\hline Ingénieurs & $-0,01$ & $0,45^{* * *}$ & $-0,35^{* * *}$ & 0,00 & $-0,38^{* * *}$ \\
\hline Docteurs & 0,02 & $-0,31^{* * *}$ & $-0,26^{* * *}$ & $-0,40^{* * *}$ & $-0,27^{* * *}$ \\
\hline
\end{tabular}

Source : auteur, à partir de l'enquête Céreq 2007 sur la Génération 2004.

Note de lecture : Différence de salaire entre emploi R\&D et emploi Hors $R \& D$ au sein du secteur $R \& D$, différence de salaire entre emploi $R \& D$ et emploi $H R \& D$ au sein du secteur $H R \& D$. Le test de student est utilisé pour déterminer la significativité des différences de moyenne.

$(* * *, * *, *$ : significatif à 1,5 et $10 \%)$.

Cette méthode économétrique nous permet de déterminer si une différence de motivation telle qu'un effet de préférence est susceptible de biaiser les résultats. À partir des paramètres des différents modèles, les salaires conditionnels et contrefactuels (cf. encadré 1 ) ont été calculés, permettant de construire les indicateurs présentés dans la partie 2. Les fonctions de gains ont été également estimées par la méthode des MCO (moindres carrés ordinaires) afin de pouvoir comparer les résultats.

ans après l'obtention de leur doctorat) ; une analyse des salaires sur le long terme est donc nécessaire pour déterminer si cet écart n’est pas temporaire. ${ }^{9}$

Les diplômés d'écoles d'ingénieurs sont employés également dans le secteur d'activité qui leur offre le meilleur salaire. $\pi_{1}$ est significativement positif alors que $\pi_{0}$ est significativement négatif dans le modèle avec les seules variables individuelles. Les ingénieurs actuellement employés dans la R\&D percevraient des salaires inférieurs s'ils se trouvaient dans le secteur hors recherche et inversement pour les ingénieurs actuellement employés dans le secteur hors recherche. Les résultats changent lorsque les variables entreprises sont introduites. En effet, $\pi_{1}$ devient non significatif, suggérant que les activités de R\&D ne sont pas plus rémunératrices que les autres activités pour les ingénieurs actuellement employés dans les activités de R\&D.

Contrairement aux docteurs, dans le cas des ingénieurs, il ne semble pas qu'en début de carrière, le secteur de $\mathrm{R} \& \mathrm{D}$ soit moins rémunérateur que le secteur hors recherche. La différence de résultat entre les docteurs et les ingénieurs peut s'expliquer par le fait que le

9. Notons que le coefficient de la variable R\&D est positif et non significatif dans le modèle estimé par les MCO. La différence de résultat entre les deux modèles n'est pas surprenante. Il semble en effet que les docteurs les « plus performants » exercent dans le secteur R\&D. Le fait de ne pas prendre en compte les biais de sélection tend donc à surestimer la rémunération dans le secteur recherche. 
secteur hors recherche constitue souvent un second choix pour les docteurs. Dans l'échantillon, seulement $18 \%$ des docteurs avec un cursus universitaire et $11 \%$ des docteurs avec un cursus antérieur en école d'ingénieurs souhaitaient travailler dans ce secteur lors de leur doctorat. Pour les diplômés d'écoles d'ingénieurs, le secteur hors recherche (production, fabrication, management...) représente un choix de carrière courant.

\section{Conclusion}

Nous avons cherché ici à identifier les facteurs déterminants de l'accès à la R\&D pour les jeunes diplômés en sciences. Tout d'abord, au sein des activités de R\&D, une différenciation apparaît dans la sélection entre les docteurs et les diplômés d'écoles d'ingénieurs. En effet, dans le cas des docteurs, le financement dont a bénéficié le docteur (CIFRE) et l'expérience en entreprise sont déterminants dans l'accès à la R\&D. Ce résultat conforte d'autres travaux (Giret, Perret, Recotillet, 2007). Pour les ingénieurs, seule l'école d'ingénieurs dont le diplômé est issu joue un rôle dans l'accès à la recherche. Les ingénieurs diplômés d'une école entretenant de nombreux partenariats en R\&D avec les entreprises ont plus de chances de devenir chercheurs et perçoivent par ailleurs des revenus supérieurs dans le secteur de la R\&D. En revanche, le prestige de l'école ne semble pas influer sur l'entrée dans la recherche. Par ailleurs, l'utilisation des réseaux ne semble pas accroître les chances d'être employé dans le secteur recherche. Seul le fait d'avoir pris connaissance d'une embauche possible par le biais de l'établissement de formation est susceptible avoir un impact sur l'entrée dans la recherche. De même, aussi bien pour les diplômés d'écoles d'ingénieurs que pour les docteurs, le partenariat avec les entreprises et les liens avec la recherche industrielle semblent déterminants. Les politiques actuelles tendent, par ailleurs, à renforcer ce type de partenariats avec la création, par exemple, des PRES (Pôles de Recherche et d'Enseignement Supérieur) ou encore des pôles de compétitivité.

Le rendement des docteurs dans la R\&D privée est supérieur à ceux des écoles d'ingénieurs. Une étude récente de Riedenger et Zaiem (2001) parvient à un résultat similaire. Celle-ci souligne également que les docteurs déposent le même nombre de brevets que les diplômés d'écoles d'ingénieurs. En revanche, la formation doctorale est moins rentable dans le secteur hors recherche. Ce résultat suggère que les entreprises ne considèrent pas les compétences acquises lors du doctorat comme transférables au secteur hors recherche. Soulignons que la plupart des docteurs dans le secteur privé exercent hors du secteur recherche. L'analyse des salaires suggère qu'en début de carrière, le secteur de la R\&D est pour eux moins rémunérateur que les autres activités ; une préférence pour la recherche, de la part des docteurs, pourrait expliquer ce résultat. Pour les diplômés d'écoles d'ingénieurs, les analyses révèlent également que, au sein des entreprises, les activités de R\&D sont peu valorisées par rapport aux autres activités. Des travaux réalisés dans d'autres pays européens corroborent ce résultat. En effet, dans le rapport "Set for success ", Roberts (2002) montre qu’au Royaume-Uni, les scientifiques employés dans des activités de R\&D 
peuvent percevoir un salaire inférieur de deux tiers à ceux de leurs confrères du secteur de la finance. En Hollande, Dupuy et Smith (2009) mettent en exergue, pour les activités de $R \& D$, une pénalité de salaire d'environ 3,5\%. Ces résultats nous conduisent à nous interroger sur la politique européenne et française actuelle visant à accroître le nombre de chercheurs dans le secteur privé. En effet, selon les estimations de Cahu et al. (2010), la nouvelle réforme du crédit impôt recherche devrait, à elle seule, entrainer un accroissement des investissements de R\&D privée de l'ordre de 0,33 points du PIB (produit intérieur brut) à l'horizon 2020 ; cela engendrerait, de la part des entreprises, un accroissement de la demande de chercheurs de l'ordre de $25 \%$ par rapport au stock actuel. La question de l'offre de diplômés en sciences et du recrutement de nouveaux chercheurs par les entreprises va donc constituer un enjeu majeur dans les années qui viennent.

\section{Bibliographie}

Audric-Lerenard A., Topiol A. (1999), "Chercheur : un métier en expansion qui permet l'embauche de jeunes diplômés ", Premières synthèses, $n^{\circ}$ 37-02, Dares, ministère de l'Emploi, septembre.

Becker F.S. (2010), "Why don't young people want to become engineers? Rational reasons for disappointing decisions", European Journal of Engineering Education, vol. 35, $\mathrm{n}^{\circ} 4$, pp. 349-366.

Beltramo J.-P., Paul J.-J., Perret C. (2000), The recruitment of researchers and the organisation of scientific activity in industry, International Journal of Technology Management, vol. 22, $\mathrm{n}^{\circ}$ 7-8, pp. 811-834.

Biddle J., Roberts K. (1994), Private sector scientist and engineers and the transition to management, The Journal of Human Resources, vol. 29, n 1, pp. 82-107.

Brown C., Linden G. (2008), "Is there a shortage of engineering talent in the US?”, Working Paper $n^{\circ} 436132$, Institute for Research on Labor and Employment, Berkeley, University of California.

Butz W.P., Bloom G.A., Gross M.E., Kelly T.K., Kofner A., Rippen H.E. (2003), “Is there a shortage of scientists and engineers? How would we know?", Rand Issue Paper $\mathrm{n}^{\circ}$ 241, Santa Monica.

Cahu P., Demmon L., Masse E. (2010), "L'impact macroéconomique de la réforme 2008 du crédit d'impôt recherche ", Revue Économique, vol. 61, n 2, pp. 319-339.

DelfgaAuw J., Dur R. (2007), "Signaling and screening of workers' motivation", Journal of Economic Behavior and Organization, vol. 62, n 4, pp. 605-624.

Duhautois R., Maublanc S. (2005), Les carrières des chercheurs dans les entreprises privées, rapport de recherche, Centre d'étude de l'emploi. 
Duhautois R., Maublanc S. (2006), "Chercheurs dans le privé : la place des docteurs ", Connaissance de l'Emploi $\mathrm{n}^{\circ} 26$, Centre d'études de l'emploi, février.

Dupuy A., Smits W. (2009), "How large is the compensating wage differential for R\&D workers?”, Discussion Papers 4194, Institute for the Study of Labor..

Fox M.F., Stephan P. (2001), "Careers of young scientists: Preferences, Prospects and Realities by Gender and Field”, Social Studies of Science, vol. 31, n 1, February, pp. 109-122.

Franzen A., Hangarten D. (2006), "Social Networks and Labour Market Outcomes: The Non-Monetary Benefits of Social Capital”, European Sociological Review 2006, vol. $22, \mathrm{n}^{\circ} 4$, pp. 353-368.

Gago J.M. (2004), Europe needs more scientists. Report by the High Level Group on Increasing Human Resources for Science and Technology in Europe, Brussels. European Commission.

Giret J.-F., Moullet S., Beduwe C., Fourcade B. (2006), « Les filières scientifiques et l'emploi ", Les dossiers Insertion Education et Sociétés n 177, ministère de l'Education nationale, Enseignement supérieur et de la Recherche.

Giret J.-F., Perret C., Recotillet I. (2003), « Les jeunes scientifiques dans le secteur privé : quel rendement de la formation doctorale ?", in Mondialisation et régulation sociale, tome 2, L'Harmattan, pp. 841-854.

Giret J.-F., Perret C., Recotillet I. (2007), « Le recrutement des jeunes docteurs dans le secteur privé ", Revue d'économie industrielle, vol. 119, pp. 85-102.

Graham J., Smith S. (2005), "Gender differences in employment and earnings in science and engineering in the US", Economics of Education Reviews, vol. 24, n³, pp. 341-354.

Granovetter M. (1995), Getting a job: A Study of Contacts and Careers. Chicago: University of Chicago Press.

Heyes A. (2004), "The economics of vocation or why is a badly paid nurse a good nurse?", Journal of Health Economics, vol. 24, n 3, pp. 561-569.

Industrie et technologies (2007), " R\&D industrielle : le $12^{\text {ème }}$ palmarès des écoles d'ingénieur ", Octobre 2007.

LAM A. (2001), "Changing R\&D organisation and innovation: Developing the new generation of R\&D knowledge workers", in The Contribution of European Socio-Economic Research to the Benchmarking of RTD Policies in Europe. DGV, Brussels: European Commission.

LAssibille G. (2001), "Earnings distribution among Spanish engineers: research vs. nonresearch occupation”, Research Policy, vol. 30, n 4, pp. 673-680. 
Lavoie M., Finnie R. (1998), “The occupational dynamics of recent Canadian engineering graduates inside and outside the bounds of technology", Research Policy, vol. 27, $\mathrm{n}^{\circ} 2$, pp. 143-158.

Levin S.G., Stephan P.E. (1991), "Research Productivity Over the Life Cycle: Evidence for Academic Scientists", The American Economic Review, vol. 81, n 1, pp. 114-132.

Loksin M. et Zurab S. (2004), "Maximum likelihood estimation of endogenous switching regression models", Stata Journal, vol. 4, n³, pp. 282-289.

Lowell B., SAlzMan H. (2007), Into the eye of the storm: Assessing the evidence on science and engineering education, quality, and workforce demand, The Urban Institute, Washington DC.

Mishagina N. (2008), Career Dynamics of Doctoral Scientists and Engineers. Working Paper, Economics Department, Queen's University, January.

Mangematin V. (2000), "PhD job market: professional trajectories and incentives during the PhD”, Research Policy, vol. 29, pp. 741-756.

Manolopoulos D. (2006), "What motives R\&D professionals? Evidence from decentralized laboratories in Greece", International Journal of Human Resource Management, vol. ${ }^{\circ} 17, \mathrm{n}^{\circ} 4$, pp. 616-647.

Moen J. (2005), "Is mobility of technical personnel a source of R\&D spillovers?”, Journal of Labor Economics, vol. 23, n 1, pp. 81-114.

Montgomery J.D. (1991), "Social Networks and Labor-Market Outcomes: Toward an Economic Analysis", The American Economic Review, vol. 81, n 5, pp. 1408-1418.

Murphy K.M., Shleifer A., Vishny R.W. (1991), “The allocation of talent: implication for growth", The Quarterly Journal of Economics, vol. 106, n² 2, pp. 503-530.

Observatoire de l'emploi scientifique (2009), État des lieux de l'emploi scientifique en France, Rapport au ministère de l'Enseignement supérieur et de la Recherche.

Reвick M.E. (2000), “The importance of networks in the market for university graduates in Japan: a longitudinal analysis of hiring patterns", Oxford Economic Paper 52, pp. 471-496.

Recotillet I. (2007), "PhD Graduates With Post-Doctoral Qualification in the Private Sector: Does it Pay Off?”, Labour, vol. 21, n³ pp. 473-502.

Riedinger N., Zaiem M. (2011), "Y a-t-il un problème d'insertion des titulaires de doctorat dans les centres de R\&D des entreprises ? "LettreTrésor-Eco, n ${ }^{\circ}$ 94, novembre.

Roberts G. (2002), "Set for success ": The supply of people with science, technology, engineering and mathematics skills, April, accessible en ligne à : http://webarchive. 
nationalarchives.gov.uk/+/http://www.hmtreasury.gov.uk/documents/enterprise_and_ productivity/research_and_enterprise/ent_res_roberts.cfm , accédé le 3/08/2010.

Saloner G. (1985), "Old Boy Networks as Screening Mechanisms", Journal of Labor Economics, vol. 3, n’3, pp. 255-267.

Simon C.J., Warner J.T. (1992), "Machmarker, Machmarker: the effect of old boy networks on job match quality, earning and tenure", Journal of Labor Economics, vol. 10, $\mathrm{n}^{\circ} 3$.

STERn S. (2004), "Do scientists pay to be scientists?", Management Science, vol. 50, n 6, pp. 835-853.

WeIss A. (1980), "Job queues and layoffs in labor markets with flexible wages", Journal of Political Economy, 88, pp. 526-538.

Zenger T.R., Lazzarini S.G. (2004), "Compensating for innovation: Do small firms offer higher-powered incentives that lure talent and motive effort", Managerial and Decision Economics, vol. ${ }^{\circ} 25$, pp. 329-345. 